This item was submitted to Loughborough's Research Repository by the author.

Items in Figshare are protected by copyright, with all rights reserved, unless otherwise indicated.

\title{
Implications on design of rapid manufacturing
}

PLEASE CITE THE PUBLISHED VERSION

PUBLISHER

Professional Engineering Publishing / @ IMechE

VERSION

VoR (Version of Record)

LICENCE

CC BY-NC-ND 4.0

REPOSITORY RECORD

Hague, Richard J.M., R.I. Campbell, and Phill M. Dickens. 2019. "Implications on Design of Rapid Manufacturing". figshare. https://hdl.handle.net/2134/4719. 
This item was submitted to Loughborough's Institutional Repository (https://dspace.lboro.ac.uk/) by the author and is made available under the following Creative Commons Licence conditions.

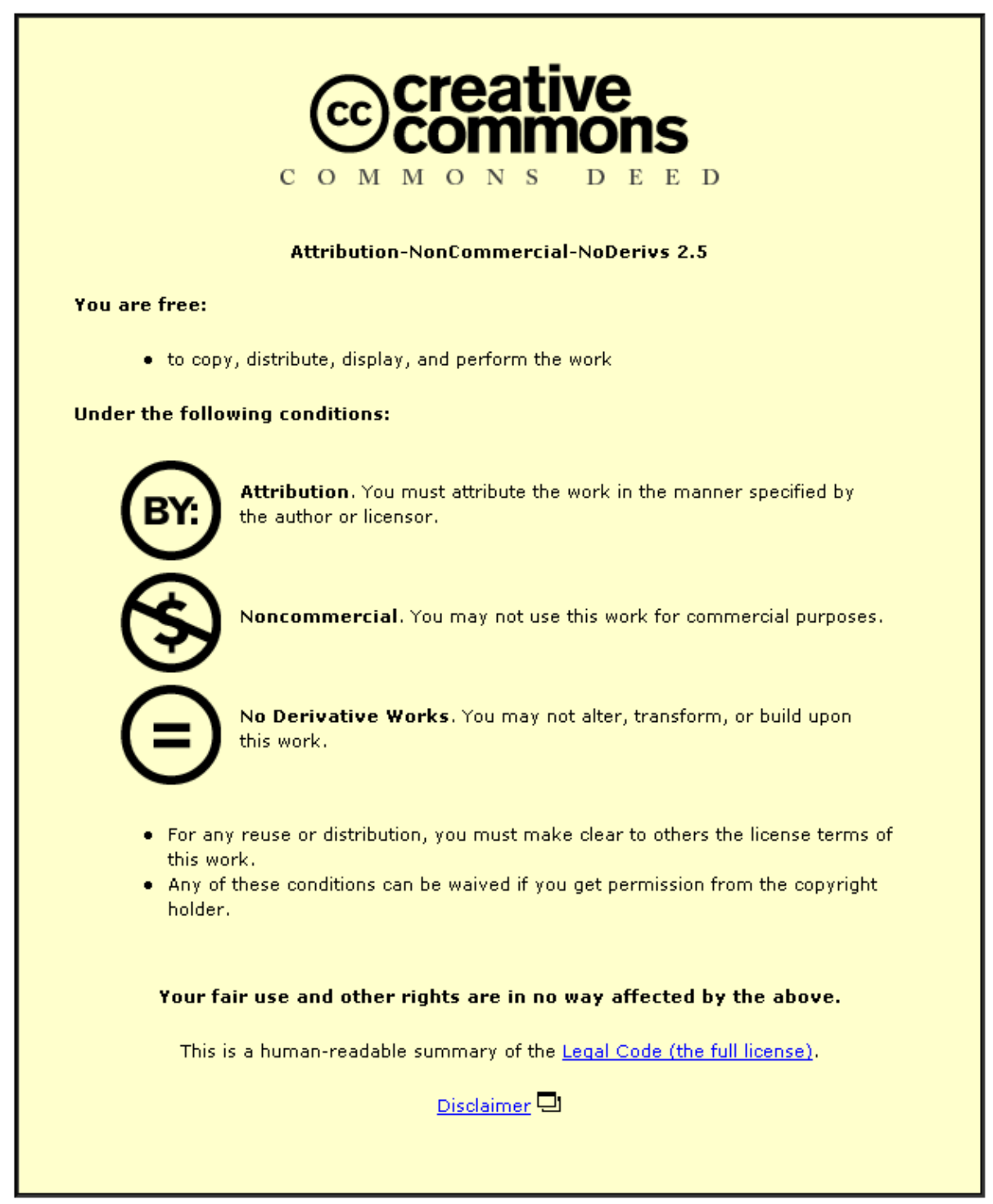

For the full text of this licence, please go to: http://creativecommons.org/licenses/by-nc-nd/2.5/ 


\title{
Implications on design of rapid manufacturing
}

\author{
R Hague ${ }^{1 *}$, I Campbell ${ }^{2}$ and $\mathbf{P}$ Dickens $^{1}$ \\ ${ }^{1}$ Wolfson School of Mechanical and Manufacturing Engineering, Loughborough University, Loughborough, UK \\ ${ }^{2}$ Department of Design and Technology, Loughborough University, Loughborough, UK
}

\begin{abstract}
During the last few decades, designers have been educated to develop designs with restricted geometry so that parts can be made easily. The revolutionary aspect of rapid manufacturing will be that geometry will no longer be a limiting factor. The introduction of rapid manufacturing will have a number of effects on design. It will be possible to have re-entrant shapes without complicating manufacturing, no draft angles, variable wall thickness, no split lines and fewer parts, leading to easier assembly and lower stock. The individual designer's method of working will change with the introduction of rapid manufacturing and also there will be changes to the overall design process.
\end{abstract}

Keywords: rapid prototyping, rapid manufacturing, design

\section{INTRODUCTION}

The aim of this paper is to discuss the implications of the introduction of rapid manufacturing (RM) on design and on designers. As this is a new subject for discussion, the authors intend that this paper be viewed as a precursor to work that is needed. Many of the ideas discussed are the subject of much current research that is being performed into this area by the authors [1].

\subsection{Definition of rapid manufacturing}

Firstly, it is important to give a definition of rapid manufacturing. The way that many products are manufactured in the future will change. The current use of moulds and dies to give items their form-be they parts for space shuttles, mobile phone covers or breakfast cereals-will, in some instances, be superseded by the emerging technologies that come under the rapid manufacturing banner. This will, in the first instance, be for low-volume products, but will also have implications for medium- to high-volume production as the RM processes improve [2].

Rapid manufacturing is evolving from the now mature rapid prototyping (RP) technologies, such as stereolithography and laser sintering (among others).

The MS was received on 25 February 2002 and was accepted after revision for publication on 10 June 2002.

* Corresponding author: Wolfson School of Mechanical and Manufacturing Engineering, Loughborough University, Loughborough, Leicestershire LE11 $3 T U, U K$.
With rapid prototyping techniques, objects are created from a three-dimensional computer aided design (CAD) model without the use of either moulding or tooling. This is achieved by taking discrete two-dimensional slices of the three-dimensional CAD file and, either through the deposition of material, the sintering or binding of powder or by selectively changing the state of an ultraviolet (UV) sensitive liquid resin into a solid using a light source, objects are built up layer-by-layer until the part is complete. Having been developed and commercialized over the last 15 years, layered manufacturing now plays an integral role in the modern product development cycle. RP processes are now routinely used for the production of functional and technical prototypes, visualization and marketing models and even for the production of 'rapid tooling' cavities, although the value of some of these so-called rapid tooling techniques is questionable.

\section{POTENTIAL OF RAPID MANUFACTURING ON DESIGN}

For years, designers have been restricted in what they can produce as they have generally had to design for manufacture, i.e. adjust their design intent to enable the component (or assembly) to be manufactured using a particular process or processes. In addition, if a mould is used to produce an item, there are therefore automatically inherent restrictions to the design imposed at the very beginning. Taking injection moulding as an example, in order to process a component successfully, 
at a minimum the following design elements need to be taken into account:

(a) Geometry

Draft angles

Non-re-entrant shapes

Near-constant wall thickness

Complexity

Split line location

Surface finish

(b) Material choice

(c) Rationalization of components (reducing assemblies)

(d) Cost

With the advent of the rapid manufacturing techniques, there is the potential for many of the current obstacles to be removed. The following sections discuss the above points and also deal with some potential problems that are likely to occur with the onset of rapid manufacturing in general.

\subsection{Geometry for 'free'}

One of the major benefits of some of the additive manufacturing processes is that it is possible to make any complexity of geometry at no extra cost. This is virtually unheard of, as in every conventional manufacturing technique there is a direct link to the cost of a component and the complexity of its design. Therefore, for a given volume of component, it is effectively possible to obtain the geometry (or complexity) for 'free', as the costs incurred for any given additive manufacturing technique are usually determined by the time to build a certain volume of part, which in turn is determined by the orientation in which the component is built.

\subsection{Design freedom}

Additionally, the advent of rapid manufacturing will have profound implications for the way in which designers work. Generally, designers have been taught to design objects that can be made easily using current technologies, this being mainly due to the geometry limitations that exist with available manufacturing processes. For parts that are moulded, this means that draft angles, constant wall thickness, location of split line, etc., have to be considered in the design. However, with the additive manufacturing technologies, these limitations are removed as it is not necessary to withdraw the part from the mould and therefore it would be possible to have re-entrant shapes without complicating manufacturing, no draft angles, variable wall thickness and no split lines. This new design freedom will place much more responsibility on the designer to think about the exact requirements of a part; with the unlimited geometry capability designers will therefore need to be much more imaginative in order to make full use of the new manufacturing processes. In the past, the processes have severely restricted designers and so they have become accustomed to designing relatively simple geometries.

The other main effect will be on the overall design process. At the moment a designer produces a rough CAD model and then a prototype is built; further design work is done until it is possible to manufacture a fully representative model. The next step may involve prototype tooling, functional testing and then production tooling and any final testing before large-scale manufacturing. If rapid manufacturing is to be used then the obvious question involves what happens to these different stages, because it will be possible to complete the CAD model and then directly manufacture the end-use part.

\subsection{Materials}

When objects are formed in moulds, they are generally formed in one homogeneous material. Even in the case of an overmoulded component, where there can be two or more homogeneous materials in one finished part, there is a definitive boundary between one material and the other. In the future, with some of the additive manufacturing processes there is the potential to mix and grade materials in any combination that is desired, thus enabling materials with certain properties to be deposited where they are needed $[2,3]$.

The overmoulding technique is a classic example of how design can be influenced by the availability of a manufacturing technique. Overmoulding allows designers, within limits, to produce parts that have added functionality and enhanced design. Indeed, the design of overmoulded components very often incorporates the different material combinations to accentuate the design to the extent that designers are able to exploit the delineation of the different materials used to produce design features as well as extra functionality. This is perfectly illustrated by the simple case of a toothbrush. This is an everyday item that will often include overmoulding to give a handle that is stiff, with an overmoulded grip and a different material at the neck to give a flexible head. This is illustrated in Fig. 1.

Additionally, there are also issues involved when using different materials in a single product, such as materials giving differences in thermal expansion or corrosion resistance. The ability to grade from one material to another could eliminate some of these problems.

Given that rapid manufacturing potentially allows the development of multiple materials to be deposited in any location or combination that the designer requires, this 


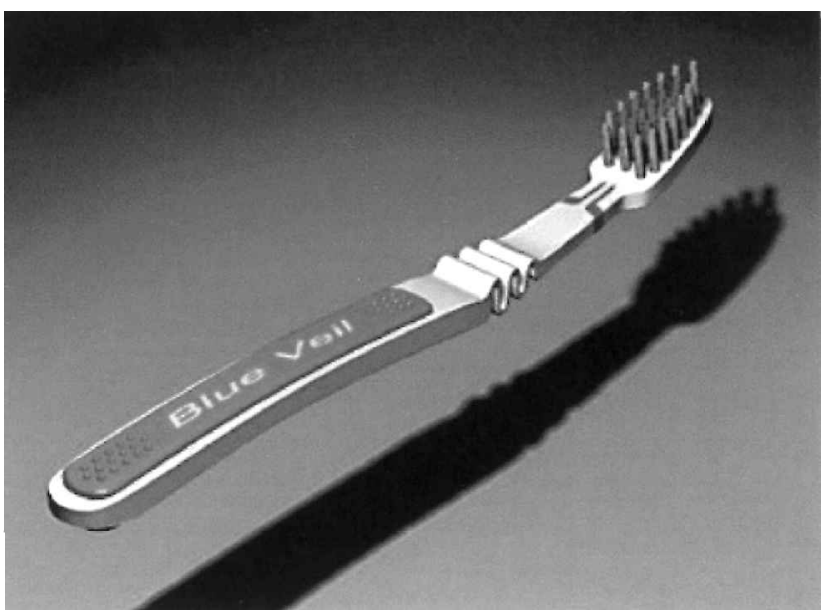

Fig. 1 Example of how overmoulding improves design and functionality

has potentially enormous implications for the functionality and aesthetics that can be designed into parts.

\subsection{Custom parts}

There is a definite trend to 'mass customization' or even total customization as people increasingly want to own items that are individual to themselves-all consumers would really like to have their own design of mobile phone (think of NOKIA Xpress-on ${ }^{\mathrm{TM}}$ covers), rather than one that looks like everyone else's. This last statement may or may not be true, but most consumers have to have mass-produced items at the moment because all phones (or whatever) are made in moulds. The additive rapid manufacturing techniques are the enabling technologies to more cost effective custommade products.

One of the biggest advantages of rapid manufacturing will be the fact that tools will not be needed for many components as it will be possible to 'print' the products directly. As it will not be necessary to mass produce parts to amortize the costs of the tooling into many thousands of components, this will lead to the potential for more custom-made parts.

The potential to produce custom-made products cheaply could prove to be lucrative to manufacturers, who will be able to charge a premium for these so-called complex custom components that are, in effect, no more expensive to produce than simple parts. In the short term, this will present tremendous profit-making opportunities for manufacturers who pursue rapid manufacturing as an alternative to conventional techniques.

However, the ability to produce cost effective custommade products will lead to the question of who will do the design work.

\subsection{Customer-driven design for rapid manufacturing}

It is thought that the rapid manufacturing technologies will split into home-based lower-cost machines (akin to the HP DeskJet of today) and more precise systems that will be company operated. However, with the ability to produce custom-made products and the advances in web-enabled software, there is the potential to include the customer within the design process to enable them to produce their component either on their home-based systems or by the OEM (or third party supplier).

So far, this paper has described the implications on design by the advent of rapid manufacturing and has tended to concentrate on the implications to existing designers. If the end user is to be involved in the design process, how can this be achieved? The following sections attempt to deal with how designs can be more custom-driven.

As discussed, rapid manufacturing potentially offers the end customer the opportunity to design, order and receive a product without leaving their home. This vision of the future has been articulated by several people, including Burns $[4,5]$. It is possible to imagine a situation where a home-based personal computer is used to design a three-dimensional object that is either produced on a three-dimensional printer beside the operator or ordered over the Internet and delivered next day. Just how close is this vision today? Certainly, threedimensional CAD packages are readily available, ordering goods over the Internet has become an everyday activity for many and overnight production of physical models is on offer by Materialise [6]. However, there are issues that still need to be tackled. Three-dimensional printers are not yet readily available to home users. Multimaterial models and production quality' surface finishes are still the topics of much research. Intellectual property and product liability problems also need to be addressed.

Leaving these issues aside, there remains the critical matter of how deeply involved customers can become in the design of a product. Expecting a member of the general public to use a conventional three-dimensional CAD system to create even simple shapes is not realistic. Even the most intuitive of menu-driven interfaces requires significant levels of training and knowledge of construction strategies. A number of options are available to overcome this constraint:

1. Restrict the design input of the user to selection from a predetermined set of alternatives.

2. Use an innovative user interface that requires little training and no construction strategy.

3. Enable the customer to work as part of the design team, e.g. in real-time collaboration with a designer.

Each of these will be discussed in turn and their potential contribution towards customer-driven rapid manufacturing will be assessed. 


\subsubsection{Restricted input}

Using parametric CAD and assembly modelling it is possible to create applications that use existing CAD models to create new product designs. The models are created by experienced designers and satisfy all the constraints of rapid manufacturing. Each model could represent a component within a particular product. An application can then be written that will allow the customer to select component options they wish to include in their 'new design'. Furthermore, some of the dimensions of the components can be varied within set limits to modify the design to meet customer requirements. This makes use of knowledge relationships within the CAD model(s). A market where this technique has already been applied successfully is in interior household design, e.g. Deco Tech 3-D Design Software [7] and Floor Plan 3D Design [8]. Applying this technique to products tailored specifically for rapid manufacturing is an area where much research is needed. An example of current research where designs are optimized to make full use of rapid manufacturing capabilities is the multimaterial work at Clemson University [9]. The user can input design requirements and the optimum multimaterial geometry is calculated automatically. CAD modelling capabilities need to be advanced to represent this type of information.

\subsubsection{Innovative interfaces}

It may be feasible to give customers access to threedimensional design through innovative interfaces where little or no training is required. An example of this is the FreeForm ${ }^{\text {TM }}$ system available from SenSable Technologies [10]. This technology enables the user to 'sculpt' a 'virtual clay' model inside a computer and in doing so can 'touch and feel' the clay as they add or remove material. It uses a haptic feedback device that gives the impression of working with a resistive medium. The geometry that is created is truly freeform and in many cases can only be produced using rapid manufacturing. The method of operation has been designed to be intuitive to the user and reduces any computational or engineering skills necessary to operate the system to an absolute minimum. This approach ensures that not only are new users 'up and running' and therefore productive within a very short period of time but they also feel far less inhibited and constrained by the technology. It can be easily envisaged that a customer could be allowed to generate new shapes, or at least modify existing designs, using this technology. An example of a novel design produced using FreeForm and the three-dimensional model produced using a Z-Corp machine are shown in Fig. 2. The stippled effect on the surface of the model was produced using 'virtual hammering'.

\subsubsection{Collaborative design}

The third technique for involving customers in the design process is through partnership with a designer. This can be readily done when the customer is brought into direct contact with the designer, e.g. in a design studio. However, if the goal is to allow the customer to stay at home then an alternative strategy must be used. Using the Internet, it is possible to connect the customer and designer using virtual conferencing, real-time imaging software (e.g. Magics Communicator [11]) and collaborative CAD software. However, designers are not always available and international time zones can cause serious difficulties. An alternative is to couple the user with a knowledge-based system that simulates an experienced designer. One such system is Cybercut ${ }^{\mathrm{TM}}$ developed at the University of California, Berkeley. This is [12]:

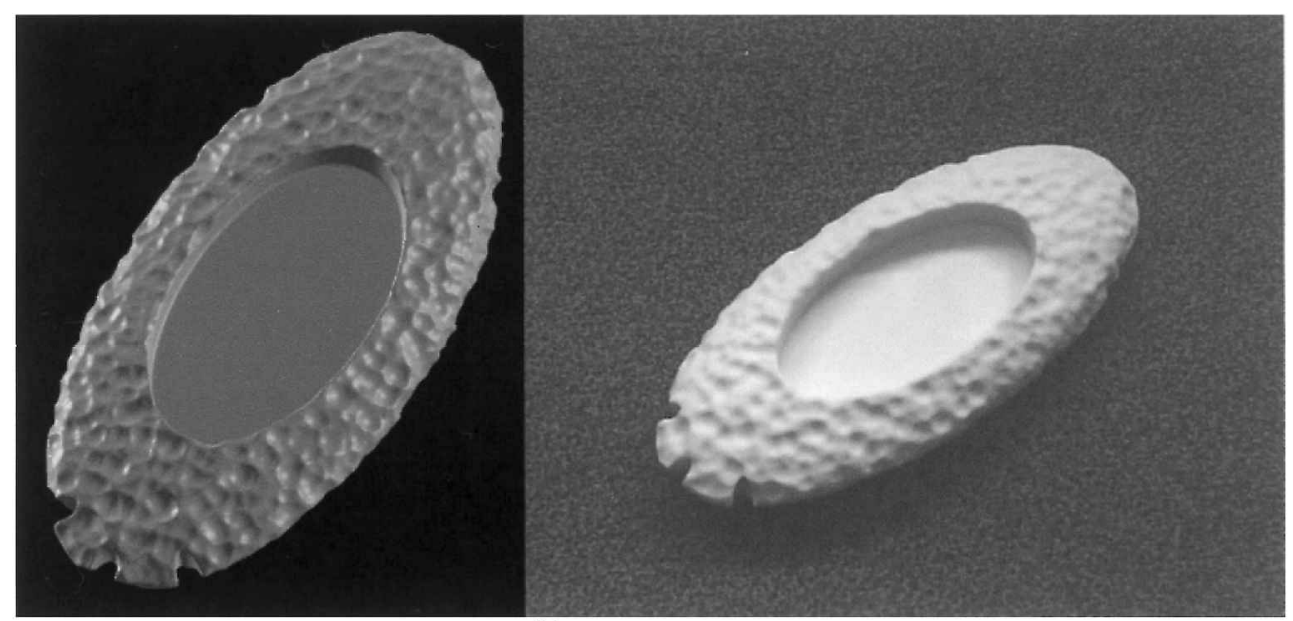

(a)

(b)

Fig. 2 (a) Virtual hammering effect created using haptic feedback; (b) resultant physical model 
a web based design-to-prototype service, allowing users to quickly design and manufacture prototypes for mechanical parts. A series of interactive web pages act as an on-line CAD tool linked to a 'computer machinist', which restricts the user to the design of 'manufacturable' parts. When the design is completed, the part specifications are sent directly to a computer controlled three axis milling machine, and fabrication can begin immediately.

It is conceivable that a similar system could be developed specifically for rapid manufacturing.

Bringing the customer further into the design process is a desirable part of rapid product development that could lead to truly customized products. The unique characteristics of rapid manufacturing make it particularly suitable for this strategy. A number of alternative techniques offer this capability and the optimum solution will vary from one product to the next. Indeed, the optimum solution may well be a combination of those discussed here.

\section{DESIGN CONSTRAINTS}

Although there will be the ability to manufacture whatever shape is required, this does not necessarily mean that there is complete design freedom. Recent work within a new UK funded EPSRC project on 'Design for Rapid Manufacture' [1] has shown that even though, in theory, it is possible to design any shape that is desired, it is still necessary to consider the incorporation of existing components with the design process.

Products are generally a combination of many components that are assembled to produce the final article. Often 'new' products incorporate components from existing proven designs and therefore the new products have to be designed accordingly to accommodate these components - this adds to the constraints in the products' design.

In addition, there is a clear need to design for assembly and maintenance. Even though there is the potential to reduce the part count by combining assemblies into fewer (more complex) individual parts, there will still be the need to assemble components such as electrical circuit boards, batteries, etc., into the final article. It is recognized that, because the objects are being built additively, there is the potential to imbed components within the build, thus obviating the 'design for assembly' issue. However, the machines required to perform these imbedding operations will be more complex and are likely to appear later than general rapid manufacturing machines and therefore the design requirements for general (single material) additive rapid manufacturing systems will need to be considered first. In addition, machines that imbed components as they build are likely to create other issues, such as how they can be recycled.

\section{OTHER POTENTIAL PROBLEMS WITH RAPID MANUFACTURING}

\subsection{Surface finish and build speed}

Significant issues that need to be overcome to enable rapid manufacturing to become more widely accepted include the surface finish (with respect to stair stepping) and build speeds, and these are somewhat interrelated. Due to the stacking or bonding of multiple crosssectional layers, the additive processes inherently produce parts that have a stair-stepped effect. This can be offset by building with thinner layers, but this can dramatically reduce the overall part build speed as there are consequently more layers to build.

\subsection{CAD}

One of the most important problems to be overcome in the future will be the limitation of existing CAD modelling systems. The new rapid manufacturing processes will be able to produce objects with different materials or microstructure throughout, but existing CAD systems cannot represent this except with a very coarse resolution or on small parts only. A few researchers in universities (such as the University of Michigan, Clemson) are investigating Voxel modelling systems. Unless great progress is made in CAD development, the situation will arise where there will be the capability of manufacturing these complex structures but no way of representing them in CAD.

\subsection{Materials}

There are two fundamental problems with materials, both of which are related to the global amount used. As the quantity of material used at present is very low compared to conventional processes, the production cost is very high. This is reflected in the sale price where, for example, stereolithography resin costs around $£ S 160 / \mathrm{kg}$ whereas ABS plastic costs $£ S 0.40 / \mathrm{kg}$. The other main problem is the limited variety of materials. As the quantity sold is low, it is more difficult to justify development of new materials.

Additionally, there is a dearth of information about even the limited number of materials that are available today-materials research is one of the keys areas at present. More reliable information is crucial to a greater uptake of rapid manufacturing as a mainstream technique.

\subsection{Recycling}

The potential to produce exotic graded materials and also to imbed components within a part as it is being 
built will present issues of recycling. How will the imbedded assemblies and exotic materials be separated for safe disposal or re-use?

At present this does not pose a particular problem as there is limited uptake of rapid manufacturing. However, if there is a larger uptake of rapid manufacturing technologies, as expected, this will become an increasing issue as the volume of parts to be disposed of will increase.

\subsection{Product liability and IPR}

With the potential for the consumer to be involved in the design of their components and also for them to be able to make them-or any other component that they download from the Internet-at home on their own rapid manufacturing machine, there are major implications for product liability and intellectual property rights (IPR) that need to be addressed.

\section{SUMMARY}

The possibilities offered by rapid manufacturing (RM) are profound. Suddenly, designers will be able to manufacture any shape that they come up with and will no longer be constrained by the necessity to produce parts in moulds. In addition, using processes such as laser sintering of dissimilar powders, RM will provide designers with new and exotic materials not available to other manufacturing processes.

At the design phase, RM allows whatever shape is desired as the mould process will no longer limit design. This means that objects can be designed with re-entrant features, no draft angles, unlimited wall thickness and increased complexity, with none of the limitations imposed by either the moulding process or the toolmaking process-as neither will be required. One of the most profound implications of RM on design will be that without the cost of tooling to amortize into the parts produced, each component can be different, potentially allowing for true mass-customization of each and every product. With developments in webenabled software and high levels of computer literacy and Internet connectivity in the home, the technologies are not far from giving the consumer the ability to modify the design of the product they desire for themselves. Although this is some way off, it is conceivable that the consumer may-for a price-want to influence the design of their new sunglasses, mobile phone casing, steering wheel grip, surgical instrument, prosthetic part or favourite kitchen utensil, etc., and then send the data back to the manufacturer to be made for them.

Rapid manufacturing will become more of a reality when the properties of the materials that are produced become more acceptable and consistent. This materials research is one of the main stumbling blocks to the adoption of these additive-manufacturing techniques for end-use parts and is the subject of much current research. In addition, the potential changes in product design and the ability for consumers to potentially build parts for themselves also brings legal and management issues such as product liability, CE marking and quality concerns such as ISO 9000-2000 conformance.

\section{REFERENCES}

1 Dickens, P. and Hague, R. Design for rapid manufacture. EPSR C Grant Reference GR/R 13517/01.

2 Anon, The solid future of rapid prototyping. The Economist Technology Quarterly, 24 March 2001, 47-49.

3 Jacobs, P.F. From stereolithography to LENS: a brief history of laser fabrication. In International Conference on Metal Powder Deposition for Rapid Manufacturing, San Antonio, Texas, 8-10 April 2002.

4 Burns, M. Automated fabrication: the future of manufacturing. Rapid Prototyping J., 1995, 1(1), 37-38.

5 Burns, M. The freedom to create. Technol. Managmt, 1995, 1(4), 157-163.

6 Materialise Next Day, http://www.materialise.com/ NextDay/nextday.html? division=NextDay.

7 Deco Tech 3-D Design, http://www.pulsara.com/.

8 Floor Plan 3D Design, http://www.imisoft.com/.

9 Fadel, G. and Saminathan, M. 3-D multi-material optimization using developed finite element code, http:// www.vr.clemson.edu/credo/.

10 FreeForm Modeling System, http://www.sensable.com/.

11 Magics Communicator, http://www.materialise.be/ communicator/.

12 Cybercut Network Manufacturing Service, http:// kingkong.me.berkeley.edu/cybercut/. 